\title{
La nueva configuración geopolítica de la cooperación al desarrollo en el continente latinoamericano: el impacto de china como nuevo donante-inversor en la región
}

Heike Clara Pintor Pirzkall hcpintor@comillas.edu

Universidad Pontificia Comillas, Madrid, España.

\section{RESUMEN}

Aunque la Unión Europea y Estados Unidos han sido donantes estratégicos en la región durante décadas, hoy susintereses geopolíticos han cambiado y América Latina ha dejado de ser prioritaria. Ante esta situación y aprovechando la retracción económica de los donantes tradicionales y por tanto una reducción de la asistencia, China está tomando el relevo, convirtiéndose en socio esencialdel desarrollo latinoamericano.Esta comunicación analizará los cambios que se han producido en las formas de cooperar y las nuevas estructuras alternativas que se están creando en el ámbito de la cooperación al desarrollo, en la que intervienen varios socios en una relación horizontal cuasi triangular, y dónde China está jugando un papel importante en una nueva configuración geopolítica del desarrollo en la regíon.
\end{abstract}

PALABRAS CLAVE: América Latina. China. Geopolítica del desarrollo. Cooperación al desarrollo. Cooperación sur-sur. Cooperación triangular. 


\section{LA COOPERACIÓN EUROPEA EN AMÉRICA LATINA}

A pesar de su posición geoestratégica entre Europa y Asia, siendo vecina de la gran potencia norteamericana, América Latina fue durante décadas el gran continente olvidado y un gigante en la sombra (Pintor, 2010). Para la mayoría de los países europeos, la cooperación al desarrollo en los años 50 y 60, fue un instrumento de sus políticas económicas, que aportaban sobre todo ayudas que potenciasen su crecimiento económico, claro reflejo de las teorías desarrollistas (Las obrasmásdestacadasen relación a la Teoría del Desarrollo son, National Bureau of Economic Research, Problems in the Study of Economic Growth, New York, 1949; United Nations, Measures for the Development of Under-developed Countries, New York, 1951; Colin Clark, The Conditions of Economic Progress, London, 1951; S.H. Frankel, The Economic Impact of Under-developed Countries, Oxford, 1953; W. W. Rostow, The Process of Economic Growth, Oxford, 1953; y W. Arthur Lewis, The Theory of Economic Growth, London, 1955. CEPAL, Informe Económico de América Latina de 1949, CEPAL, 1950., Prébisch, Raúl; Nueva política comercial para el desarrollo, México, D.F., Fondo de Cultura Económica, 1964. Furtado, Celso; Desarrollo y subdesarrollo, Buenos Aires, Eudeba, 1964.Ocampo, J.A; "New economicthinking in LatinAmerica", Journal of Latin American Studies, vol. 22, parte 1, Cambridge, Cambridge UniversityPress, 1990) imperantes en ese momento. Con la ayuda de la asistencia financiera europea se llevaron a cabo muchos proyectos de infraestructuras y telecomunicaciones para incrementar el nivel de competitividad de la industria latino americana y proyectos agrícolas, aunque el continente nunca fue una región prioritaria en las políticas de cooperación y los presupuestos por tanto siempre fueron bastante limitados. Es necesario recordar que la región sufrió la dominación de Estados Unidos durante décadas, limitando las posibilidades de acción por parte de otros países, que no tenían interés en desafiar al gigante norteamericano. La cooperación norteamericana tuvo siempre un comportamiento singular y estaba fuertemente condicionada por la rivalidad a todos los niveles frente al bloque soviético (PINTOR 2010).La revolución cubana por ejemplo, obligó al gobierno norteamericano a tomar medidas para contrarrestarla,en forma de importantes paquetes de ayuda, con el fin de parar el avance del Comunismo y controlar la polarización ideológica de la región.

Durante la Guerra Fría, Francia y Gran Bretaña, antiguas potencias coloniales, centraron sus políticas de cooperaciónen la promoción comercial,firmandoacuerdos de intercambio comercial y de cooperacióncon sus antiguas colonias, manteniendo el vínculo paternalista "político-cultural"y accediendo con facilidad a importantes materias primas necesarias para su propio desarrollo industrial, estableciéndose una relación de mutua necesidad conocida comopreferencia comercial recíproca(Unceta y Yoldi, 2000). El mantener una relación privilegiada con las antiguas colonias, fue también un objetivo prioritario de la recién fundada CEE, hoy Unión Europea y se vio plasmado en la firma de importantes acuerdos comerciales comola convención de Yaundé I (1963-1969) y Yaundé II (1969-1975) o el Tratado de Lomé. En el caso de Alemania, la cooperación al desarrollo fue desde sus inicios una herramienta de "rebranding"(branding es un anglicismo empleado en mercadotecnia que hace referencia al proceso de hacer y construir una marca (en inglés, brandequity) mediante la administración estratégica del conjunto total de activos vinculados en forma directa o indirecta al nombre y/o símbolo (logotipo) que 
identifican a la marca influyendo en el valor de la marca, tanto para el cliente como para la empresa propietaria de la marca, (RAE, 2016). muy útil para promocionar la nueva imagen del Estado alemán y conseguir así el reconocimiento internacional. La cooperación al desarrollo fue también un elemento que apoyaría -y sigue apoyando- el esfuerzo exportador alemán en todo el mundo. En América Latina, la cooperación alemana en este primer períodose caracterizó por su enormedispersión (Pintor, 2010) y falta de coherencia sin un programa específico, sino más bien acciones basadas en la improvisación (Bodemer, 1984) y fuertemente condicionadas por las necesidades políticas y comerciales del gobierno alemán. El interés central de Alemania era mejorar sus relaciones bilaterales con el mayor número de países posibles para poder acceder a materias primas a bajo precio que potenciase su despegue económico post-bélico conocido como milagro económico alemán o"Wirtschaftswunder". En todo caso la ayuda al desarrollo fue durante estas primeras décadas de carácter bilateral y fuertemente condicionada, garantizando un mayor control por parte de los gobiernossobre su uso (PINTOR, 2010).

\section{CAMBIOS GEOPOLÍTICOS EN LA COOPERACIÓN AL DESARROLLO Y EL FINAL DE LA GUERRA FRÍA}

Las conclusiones del informe Pearson y posteriormente la Comisión Brandt (Fischer 1981) tendrán un impacto muy importante en el enfoque que se dará a partir de la década de los ochenta en las políticas de cooperación al desarrollo. Aunque fomentar el crecimiento económico seguirá siendo un elemento prioritario para el desarrollo es necesario incluir otros elementos sí se quiere erradicar la pobreza. Es por tanto que las políticas de cooperación empezarán a centrarse no sólo en potenciar el crecimiento, sino garantizar la redistribución del ingreso con el objetivo de satisfacer al menos las necesidades básicas de las personas y potenciar así el desarrollo humano. Los proyectos se centrarían a partir de ahora en la creación de infraestructuras que faciliten la accesibilidad a servicios básicos cómo agua, sanidad, educación etc. con la esperanza de potenciar en el futuro el rendimiento productivo de los más desfavorecidos. Las ayudas a los países en vías de desarrollo habían estado en la mayoría de los casos condicionadas por intereses geoestratégicos de los propios donantes, limitando el crecimiento estable y sostenible. En muchos casos, la ayuda se concedía para obtener ventajas políticas, o bien para fomentar las exportaciones del país donante mediante las ayudas ligadas o condicionadas (Unceta y Yoldi, 2000) y los receptores la usaban para financiar sus guerras o llenar los bolsillos de gobernantes corruptos, cómo ya se mencionó en el apartado anterior.

El fracaso de las políticas de cooperación y la crisis que viven muchos países donantes, van a tener un impacto también en la sociedad desarrollada, que reclamará un nuevo orden mundial más justo y equitativo. El sistema de Bretton Woods se desintegra y un nuevo sistema económico liberal fue gestándose. Ese cambio del orden económico y la crisis del petróleo provocarán un período de fuerte endeudamiento que tendrá un efecto especialmente duro en América Latina. El avance del liberalismo económico provocó un impacto negativo en la cooperación, quedando esta relegada a un segundo plano. No será hasta la desintegración de la Unión Soviética y la reconfiguración geopolítica del mundo, en que la cooperación vuelve a estar en auge. De una confrontación este-oeste, 
nos encontraremos en la década de los noventa con una nueva reconfiguración y polarización norte-sur con la atención centrada en los países del este de Europa. Ante la disminución de la ayuda oficial al desarrollo por parte de los donantes o su viraje al este y una creciente "fatiga de la ayuda", muchos países pobres se verán abandonados y desplazadosespecialmente en América Latina. (Griffin, 1991). Ante el aumento también durante este período de conflictos internacionales, guerras civiles, catástrofes naturales y crisis sociales se incrementarán las aportaciones de ayuda humanitaria y de emergencia en detrimento de la ayuda oficial al desarrollo.El Comité de Ayuda al Desarrollo (CAD) y la Organización para la Cooperación y el Desarrollo Económico (OCDE) realizaron una serie de propuestas enfocadas en mejorar la eficacia de la cooperación al desarrollo ante el aumento de las disparidades y los efectos negativos de los recortes en cooperación. Provocaron así el inicio del debate a todos los niveles. Reflejo de ello fue la presentación del documento Reshapingthe 21st.Century (CAD, 1996) que indica claramente la necesidad de crear unos objetivos comunes del desarrollo. Los nuevos debates del desarrollo y sobre su eficacia, se verán institucionalizados en una serie de Cumbres auspiciadas por Naciones Unidas que darán un nuevo enfoque a la cooperación al desarrollo y a sus funciones. Los nuevos objetivos del desarrollo estarían centrados en dos elementos clave: el desarrollo humano y el desarrollo sostenible(Bodemer, 2001), alejándose cada vez más de las tendencias economicistas del pasado y acercándose a una visión más humana, donde se fomenten las capacidades (Nussbaum, 2012) y una dimensión médio ambiental.Sobre esta base se logró la celebración de la Cumbre del Milenio (2000) y a la posterior Declaración del Milenio cómo puntos de partida de los que serían los Objetivos del milenio (ODM).

En América Latina los ajustes económicos, el incremento de la pobreza yla marginalidad, obligarona los países desarrollados a centrar sus políticas de cooperación en reducir las fuertes disparidades que sufría la región. La Unión Europea (UE) jugó un importante papel en la década de los 90 y se convirtió en el principal donante y segundo mayor inversor tanto en Europa del Este cómo en América Latina, especialmente después de que la cooperación al desarrollo se convirtiese en una política común gracias al Tratado de Maastricht en 1993. El comercio de bienes se incrementó en la última década hasta alcanzar los €202 mil millones (6,3\% del comercio total de la UE y el $13 \%$ del comercio de América Latina y el Caribe(Servicio Europeo de Acción Exterior 2014). La inversión extranjera directa de la UE en América Latina y el Caribe fue mayor que la destinada a Rusia, China e India, juntos. La Unión Europea ha establecido un fondo de $€ 2700$ millones en ayuda al desarrollo dentro del marco del Instrumento de Cooperación al Desarrollo para América Latina 2007-13(Servicio Europeo de Acción Exterior, 2014), prestando especial atención a temas vinculados a la cohesión social y la integración regional, así como al refuerzo de las instituciones públicas, el desarrollo de un espacio común de educación superior UE-AL, y el fomento del desarrollo sostenible. El principal instrumento jurídico de la cooperación de la Unión Europea con América Latina es el Instrumento de Financiación de la Cooperación al Desarrollo (ICD) con importantes dotaciones financieras a los ACP y a América Latina en una nueva distribución en programas geográficos. Con la visión de que la integración regional era esencial para la estabilidad e integración de América Latina en el nuevo orden internacional, Europa ha apoyado y financiado un sinfín de 
proyectos en los espacios regionales: Mercosur, el Sistema de la Integración Centroamericana (SICA) o la Comunidad Andina (CEPAL, 2013).

A pesar del enorme impulso dado a la cooperación entre América Latina y Europa en décadas anteriores, hoy los intereses de ambas regiones han cambiado y la crisis económica actual ha reducido las ayudas al continente latinoamericano por parte del viejo continente. Ante esta situación los países latinoamericanos en alza han buscado nuevos socios en otras regiones. Europa se encuentra en recesión, limitándose las inversiones y reduciéndose los presupuestos de cooperación al desarrollo mientras que el crecimiento económico en el continente latinoamericano es no sólo de las grandes potencias como Brasil o México, sino también de países de renta media como son Colombia o Perú (Gratitus, 2013). Este vacío dejado por la UE ha sido aprovechado especialmente por China, que se ha convertido en un importantísimo inversor. Además, países como España y Portugal ante la situación crítica de sus economías, han pedido ayuda a América Latina y a China. Un ejemplo claro de cómo puede haber una reversión y cómo el papel de los países latinoamericanos y su posicionamiento puede cambiar en un plazo relativamente breve.

\section{CHINA: UN NUEVO “DONANTE-INVERSOR” EN AMÉRICA LATINA}

Durante siglos, los galeones españoles navegaban entre Acapulco y Manila, transportando productos asiáticos y americanos haciendo de puente entre ambas regiones.En el siglo XIX, decenas de miles de chinos llegaron a las costas americanas para trabajar en la construcción de canales y ferrocarriles, plantaciones de azúcar y minas de guano y salitre (Moreno, 2014). En el siglo XX arribaron inmigrantes para la construcción del ferrocarril, hoy la cooperación entre ambas regiones se basa especialmente en el intercambio comercial, agropecuario, minero pero también en la cooperación tecnológica, académica y cultural con un fuerte incremento en los últimos años.

Los primeros contactos entre China y América Latinafueron de carácter políticoy se sitúan en la década de los sesenta, época de fuertes tensiones ideológicas en plena Guerra Fría. América Latina se encontraba bajo la subordinación de Estados Unidos y China estaba centrada en sí misma en plena revolución cultural y aislada del mundo.Cabe denotar que a pesar de su aislacionismo el gobierno chino apoyó movimientos revolucionarios de corte comunista en el Tercer Mundo cómo fue el caso de Cuba (1960), que restableció relaciones diplomáticas con China a principio de los años sesenta y eso le valió una serie de préstamos importantes sin intereses además de acuerdos comerciales preferenciales (CEPAL, 2008), a diferencia otros países del continente latinoamericano que viraron mayoritariamente hacia el norte. Cómo contraposición el resto de América Latina abrazó el programa Alianza para el Progreso durante los años 1961 a 1970. Se proyectó una inversión de unos 20.000 millones de dólares, en su mayoría de agencias de cooperación americanas y agencias financieras multilaterales como el Banco Interamericano de Desarrollo el Fondo Monetario Internacional y el Banco Mundial y el sector privado, canalizados a través de la Fundación Panamericana de Desarrollo (Agudelo, 1966). El objetivo principal de la Alianza para el Progreso era mejorar la 
productividad agrícola e industrial, modernizar las telecomunicaciones y mejorar la calidad de vida de las personas mejorando infraestructuras cómo hospitales, colegios, alcantarillado...etc. Realmente este proyecto fue creadopor el presidente Kennedy como instrumento de contención contra el avance del Comunismo en la región y cómo propaganda para contrarrestarel éxito de la revolución cubana.

El proceso de reformas iniciadas en 1978 con DengXiaoping sólo era posible con una apertura al exterior. Esta nueva "economía socialista de mercado" provocó profundas transformaciones internas y un nuevo posicionamiento de China en el mundo (Cesarín yMoneta, 2005). Por tanto y en consonancia con su política exterior aperturista, en la década de los setenta China restableció relaciones diplomáticas con algunos países latinoamericanos como Chile (1970) o Perú (1971), dotando con ayuda humanitaria y asistencia financiera al Istmo Centroamericano devastado por innumerables catástrofes naturales (Oficina de Información del Consejo de Estado Chino, 2011). Es obvio que durante la Guerra Fría, China apoyaría a países de su esfera de influencia, cómo fue el caso de las primeras ayudas en los años cincuenta a Corea y Vietnam en plena efervescencia bipolar. Cinco años más tarde la ayuda se extendió a otros países socialistas, especialmente a países del continente africano, en muchos casos colonias recientemente independizadas. En los años sesenta, en pleno proceso de descolonización, el gobierno chino centró la cooperación al desarrollo principalmente en ayuda financiera y asistencia técnica y a finales de los setenta China buscó nuevas formas de cooperación mucho más flexibles y tendió a una mayor diversificación, además se centró especialmente en los países menos adelantados y empezó a realizar estudios sobre el impacto de la ayuda en estos países, racionalizando y mejorando la gestión de los recursos (Oficina de Información del Consejo de Estado Chino, 2011).

En la década de los ochenta, será especialmente importante la transformación económica en la propia China y su apertura al comercio internacional. Prueba de ello es su incorporación a organizaciones internacionales como el Fondo Monetario Internacional (FMI), el Banco Asiático de Desarrollo o la Organización Mundial del Comercio. Las relaciones comerciales se convertirán desde este momento en el eje central de las relaciones de China América Latina, con un interés recíproco por ambas partes. La cooperación china se gestionará y supervisará desde el Ministerio de Finanzas con la aprobación del Consejo de Estado y el Congreso.Se inicia con una reorientación de las políticas de cooperación y una mayor apertura hacia el exterior por parte de China. Para América Latina este cambio permitió un mayor involucramiento de China en proyectos de capacitación tecnológica en el sector agrícola, energético y sanitario principalmente que fueron complementándose con ayudas de carácter humanitario con equipos de asistencia médica y aportaciones al Banco Interamericano de Desarrollo, la Comunidad Andina y la OEA entre otros (Centro de Prensa CEPAL 2014).

Los mayores cambios surgen a raíz del despegue económico de China en la década de los noventa y el enorme desarrollo que el país experimenta. En 1993 el Gobierno Chino crea un Fondo de Ayuda Externa para las JointVentures (acuerdo comercial de inversión conjunta a lo largo plazo entre dos o más empresas) y proyectos de desarrollo (Cesarín yMoneta, 2005). En el año 2000 se constituye el Foro de Cooperación China-África (FOCAC), que se convirtió en una importante 
plataforma de dialogo entre China y los países africanos confirmándose como un efectivo mecanismo de cooperación. El cambio más importante en este período en relación a la cooperación al desarrollo es el tema de los plazos, ya que se pasa de ayudas generalmente a corto plazo a nuevos proyectos a plazos más largos, que implica una mayor corresponsabilidad por parte de China y los receptores (Oficina de Información del Consejo de Estado Chino, 2011).Desde el 2004 los recursos financieros de China para la ayuda externa se incrementaron rápidamente, además China participó más en conferencias internacionales y regionales reforzando su imagen exterior.

En el 2010 y después de importantes cambios tanto a nivel político como económico, el Gobierno Chino organizó la Conferencia Nacional sobre Ayuda Externa para definirlos nuevos objetivos de la cooperación al desarrollo.En la actualidad las relaciones entre China y muchos países latinoamericanos han dado un giro inesperado muy positivo. Por un lado China tiene acceso directo a los recursos naturales de la región, que necesita para su propio desarrollo, y América Latina consigue un socio y prestamista. Desgraciadamente el incremento y aceleración de los flujos comerciales entre China y los países emergentes latinoamericanos está causando también un impacto muy negativo en el medio ambiente y en la comunidades locales cercanas a las minas y zonas de explotación (Watts, 2013), además en muchos casos no se ha integrado en esta ecuación, el concepto de desarrollo sostenible con unas consecuencias devastadoras para el futuro, si no se toman medidas al respecto. Además de las inversiones en América Latina, China también ha aportado recursos para un mayor desarrollo de la región por medio de proyectos conjuntos. China consiguió en el año 2011, el 30\% del acervo mundial de reservas oficiales internacionales, casi la mitad de todas las reservas de las economías emergentes y en desarrollo. Esto ha sido posible debido a los importantes superávits comerciales y en cuenta corriente que China ha ido registrando en los últimos años (CEPAL, 2012). El modelo chino ha ido adaptándose a los cambios impuestos por la globalización "maridando" el capitalismo con el comunismo de forma peculiar. Esto le permite ampliar sus horizontes y buscar un nuevo posicionamiento a nivel mundial, una vez que ha finalizado el mundo bipolar. En relación a América Latina, el inicio del nuevo milenio impulsó las relaciones entre ambas regiones como nunca antes se había visto, ya que también para América Latina las cosas habían cambiado en buena medida.

Después de haber estado durante décadas a la sombra de los Estados Unidos. Hoy América Latina se ha convertido en un importante socio económico y aliado político para muchos países. Después de los estragos producidos por la deuda externa y los ajustes impuestos por el Fondo Monetario Internacional en la denominada década perdida, América Latina goza hoy de economías más flexibles y renovadas. Tanto es así, que las economías latinoamericanas como la economía china han afrontando con muy buenos resultados la crisis económica actual, mucho mejor que las potencias europeas y definitivamente mejor que Estados Unidos. El declive económico europeo y la falta de interés de Estados Unidos por la región, ha creado un vacío que ha sido aprovechado de forma muy oportuna por China, especialmente en las relaciones comerciales.

En 1990, el 60 por ciento del comercio de América Latina fue con los Estados Unidos y sólo el 10 por ciento fue a Asia, hoy sólo el 40 por ciento del comercio es con los Estados Unidos y el 20 por ciento es con Asia. Lograr estos crecientes 
niveles de interlocución con China, son un importante contrapeso a la influencia de los Estados Unidos en la región (BID), las cifras lo corroboran claramente. En el gráfico a continuación (figura 1) puede observarse el incremento de la participación china y de otros países de Asia-Pacífico en el comercio con América Latina y el Caribe, indicando las exportaciones e importaciones totales de cada país.

Figura 1 - Comisión Económica para América Latina y el Caribe, CEPAL (2011).

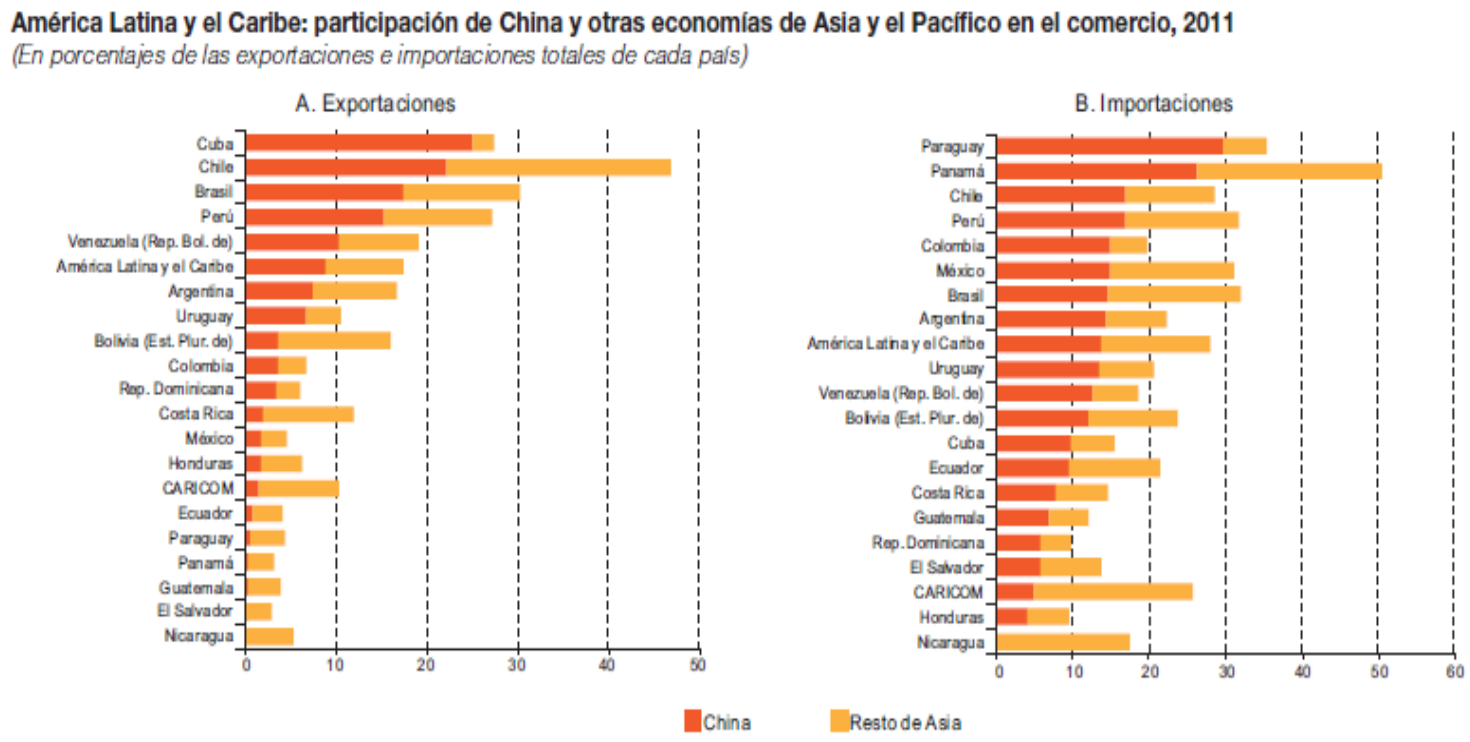

Fuente: Comisión Económica para América Latina y el Caribe (CEPAL), sobre la base de información oficial de los paises y del Fondo Monetario Internacional, Direction of Trade Statistics (DOTS).

Los países asiáticos no cooperan con todo el continente sino que están concentrando sus inversiones principalmente en los países emergentes. China es hoy el mayor socio comercial de Brasil y Chile, y el segundo de Argentina, México, Perú y Venezuela. Habiendo firmado recientemente tratados de libre comercio con Chile, Perú y Costa Rica, los cuales han promovido aún más el desarrollo de las relaciones comerciales y económicas con estos países. Por el contrario, el resto de Centroamérica y el Caribe mantienen un mayor vínculo con Estados Unidos por cercanía geográfica y causas históricas (Gratitus, 2013).La reciente firma de tratados comerciales como el Tratado de Libre Comercio de América del Norte (TLCAN) o North American Free TradeAgreement(NAFTA) y el Tratado de Libre Comercio (TLC) o Central America Free TradeAgreement (CAFTA) los vincula aún más a Norte América.

Cómo hemos mencionado antes, las inversiones y cooperación china están focalizadas sólo en países muy específicos, no existiendo una estratégica global para todo el continente, sino estrategias individuales de inversión directa en recursos naturales con países "mineros" del cono sur y México. Esto se debe a que estos países tienen enormes reservas de minerales y petróleo indispensables para el motor de la economía china. América Latina es la segunda región del mundo con mayores reservas petrolíferas, tiene reservas de litio, plata, cobre, estaño, y enormes reservas de agua y madera de sus bosques tropicales. Además es necesario tener en cuenta, que América Latina se ha convertido en una región 
aún más atractiva desde que se intensificaron los conflictos en Oriente Medio y África, dónde la extracción de los recursos no sólo es dificultosa sino está siendo muy arriesgada a causa de los fuertes enfrentamientos entre los gobiernos y las guerrillas sobre el control de los recursos. Entre 2005 y 2010, los bancos estatales chinos prestaron 75000 millones de dólares a la región, más que los préstamos del Banco Mundial, el Banco Interamericano de Desarrollo y el Banco de Exportaciones e Importaciones de los Estados Unidos juntos (BID, 2014), lo que indica claramente la importancia de la región y confirma la política expansionista china. En el gráfico a continuación (figura 2) puede verse cómo las inversiones directas al continente latinoamericano fueron de un $13 \%$ a finales del 2011, las de mayor cuantía fuera de Asia (71,4\%). A pesar de la cercanía geográfica las inversiones chinas en Oceanía no alcanzaron el 3\%, datos similares observamos en relación a Estados Unidos (3.2\%) o África (3.8\%) (ECLAC, 2012).

Figura 2 - EconomicCommissionforLatinAmerica and the Caribbean (ECLAC), Ministerio de Comercio de China (2012).

China: stock of outward foreign direct investment by region, end-2011 (Percentages)

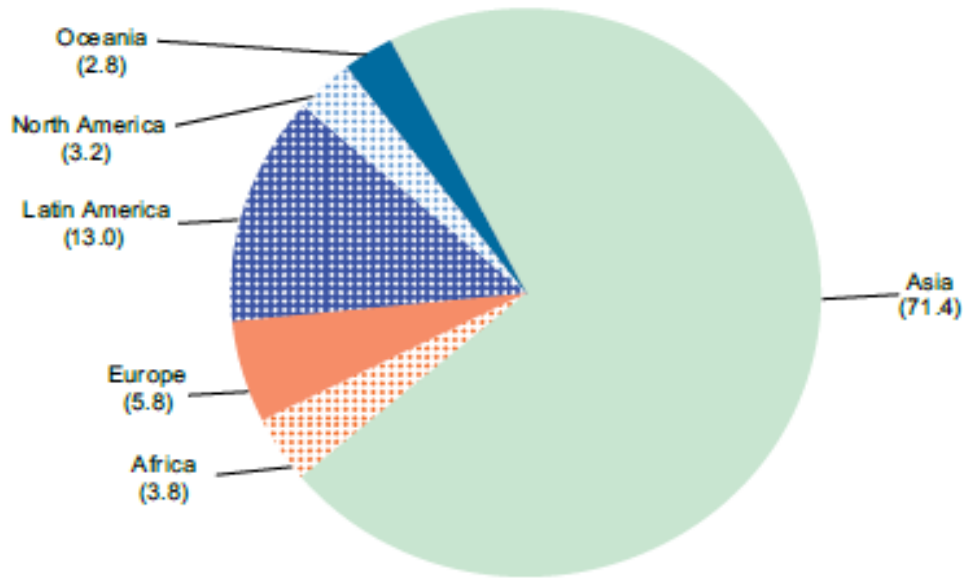

Con la llegada del nuevo milenio, China refuerza aún más los lazos con América Latina, especialmente con Brasil, creando de una serie de Foros de Cooperación Económica Comercial. El primero tuvo lugar en el 2004 y le siguieron otros en el 2005, 2007 y 2010. La "XII Cumbre de Cooperación Económica AsiaPacífico" (APEC) se vio reforzada con la visita del presidente chino Hu Jintao a Brasil, Chile, Cuba y Argentina, evento de características históricas ya que no se producía una visita desde 1981. Antonio Prado, Secretario Ejecutivo Adjunto de la Comisión Económica para América Latina y el Caribe en el Primer Foro de Ministros de Agricultura China-América Latina y el Caribe finalizado el 9 de junio 2013 en Beijing, destacó que existen "complementariedades" entre China y América Latina en el tema agrícola por lo que se realizarán actividades conjuntas en este sector y en los sectores de investigación y promoción comercial (CEPAL, 2012).

4 NUEVAS FORMAS DE COOPERACIÓN Y EL PAPEL DE CHINA EN AMÉRICA 
Lo que está claro, es que los viejos modelos de cooperación bilateral se han quedado obsoletos. La integración regional en América Latina ha potenciado nuevas formas de cooperación mucho más beneficiosas, eficaces y justas (Pintor,2013). La cooperación sur-sur (la Cooperación Sur-Sur (CSS) está basada en relaciones directas y horizontales entre países que enfrentan problemas comunes y que tienen como propósito superar, a partir de esfuerzos conjuntos, los desafíos del desarrollo. La Cooperación Sur-Sur promueve el desarrollo pleno de nuestros países, a través de mecanismos como: el intercambio comercial, el intercambio de experiencias exitosas, y la inclusión. La CSS y Triangular, y en consecuencia los proyectos que en esta materia se instrumentan en América Latina y el Caribe se caracterizan por sus principios básicos de solidaridad, complementariedad, igualdad, no condicionalidad y respeto de la soberanía. (SELA, 2012) y la cooperación triangular son definitivamente la nueva apuesta de la mayoría de países latinoamericanos, que quieren ser copartícipes trabajando en una alianza con sus nuevos "socios" y no con los donantes tradicionales del pasado. Este tipo de cambios en la cooperación al desarrollo claramente han sido posibles gracias a los importantes cambios a nivel integrativo (Haldenwang, 2005) que se han producido en los últimos años.En la actualidad, hablar de integración internacional o integración regional, se refiere a un proceso que tiene lugar entre dos o más Estados en una escala geográficamente limitada y en un plano inferior al de la integración global. La integración regional constituye un esfuerzo o movimiento convergente realizado por parte de los Estados, tendente a la obtención de objetivos comunes, mediante la armonización o unificación no sólo de políticas económicas y financieras, de regímenes monetarios, sino también de políticas socio-culturales y legislativas. Este logro no es posible si la asociación carece de sentido o de finalidad así como de unos objetivos claramente definidos tanto a nivel político como económico (IBEROASIA, 2013). Por ejemplo, en el sistema de las Naciones Unidas, Brasil y China representan actualmente la mayor fuente de financiación de la cooperación Sur-Sur (ILO, 2012).

En el caso latinoamericano la integración regional es una realidad visible, con unos beneficios claros. Las iniciativas de la integración regional en la última década se han basado casi exclusivamente en dos planteamientos. Uno político (unión aduanera) y otro comercial (zona de libre comercio). Para el primer modelo fue determinante la iniciativa estadounidense de un Área de Libre Comercio de las Américas (ALCA). El segundo enfoque está representado por el Mercosur, con Argentina, Brasil, Paraguay y Uruguay como países miembros. En la segunda mitad de los años noventa hay que resaltar también dos grandes iniciativas de integración infraestructural: el Plan Puebla-Panamá (PPP), para Centroamérica, y la Iniciativa para la Integración de la Infraestructura Regional Suramericana (IRSA), para el Cono Sur (Haldenwang2005). Esta integración también es visible en una serie de acciones conjuntas como por ejemplo el Foro de intercambios ThinkTanks, dónde América Latina ha creado una estrategia conjunta para coordinar así de forma más eficaz los intercambios sociales culturales y económicos con China alejándose de las relaciones bilaterales clásicas. También se han realizado propuestas para crear un encuentro con Jefes de Estado africanos y China además de una mejor logística y coordinación entre embajadas latinoamericanas en Asia. El director de la división de comercio internacional de la Comisión Económica para América Latina y el Caribe (CEPAL), Osvaldo Reyes, manifestó la necesidad de "aprender a pensar juntos" y construir una plataforma de diálogo para desarrollar las relaciones con China y dar 
respuesta a la gestión del país asiático (Observatorio Iberoamericano AsiaPacífico 2010).

En 2009 China anunció, junto con Argentina y Brasil, que los bancos centrales de estos dos países establecerían un mecanismo de canje de monedas. China ha propuesto el establecimiento de un fondo de cooperación sino-latinoamericana y las instituciones financieras chinas que han contribuido con un fondo inicial de 5.000 millones de dólares. El Banco de Desarrollo de China ha tomado la iniciativa al crear préstamos especiales valorados en 10.000 millones de dólares para promover la cooperación en la construcción de infraestructuras en América Latina (Observatorio Iberoamericano Asia-Pacífico 2010). Además de Brasil y Argentina, los países más beneficiados son Chile, Venezuela, Ecuador y México.

En relación a la cooperación con Brasil en el 2011, China firmó un acuerdo (Plan de Acción Conjunta 2010-2014) en el cual se establecieron las bases para la construcción de un laboratorio de investigación agrícola y la se potenció la cooperación científica en general. En el 2012 se iniciaron las obras para la construcción del laboratorio que centrará sus investigaciones en temas relacionados con la ganadería, veterinaria agro-ecología y temas medioambientales (Observatorio Iberoamericano Asia-Pacífico 2010). Brasil también se ha visto beneficiada por acuerdos energéticos de transmisión de información tecnológica para un proyecto de generación hidroeléctrica que producirá electricidad para regiones alejadas del país y aumentará la capacidad eléctrica para suministrar a las grandes ciudades brasileñas ayudando a desarrollar aún más sus industrias e infraestructuras. En la visita del presidente chino Hu Jintao a Brasil también se firmaron importantes acuerdos económicos y de defensa, creando una asociación bilateral estratégica entre ambos países. El potencial comercial existente entre ambos países es inmenso, especialmente en el sector energético y minero. Ambos países quieren promover proyectos de cooperación conjunta tanto de empresas brasileñas en China, como chinas en Brasil para el suministro de petróleo y para el desarrollo de nuevas fuentes energéticas renovables como son los biocombustibles, la biomasa o la energía solar o eólica. Para China es esencial el tema de la explotación de los minerales, y por tanto se ha comprometido a invertir en la mejora de las infraestructuras mineras de Brasil para agilizar la explotación y procesamiento de los recursos naturales en la zona. A continuación en la Figura 3 podemos observar la periodicidad de los viajes de autoridades chinas a países latinoamericanos. Entre los más favorecidos además de Brasil nos encontramos con Argentina, Uruguay, Venezuela, México, Costa Rica y Cuba. 
Figura 3 - Sitio Oficial del Gobierno de China (2013)

\begin{tabular}{|c|c|c|c|}
\hline Fecha & Autoridad china & China a América Latina & América Latina a China \\
\hline 2001 & Jiang Zemin & $\begin{array}{l}\text { Chile, Argentina, Uruguay, Brasil, Venezuela } \\
\text { (República Bolivariana de), Cuba }\end{array}$ & Perú, Chile, México, Venezuela (República Bolivariana de) \\
\hline 2002 & Jiang Zemin & México (visita no oficial) a & Ecuador, Uruguay \\
\hline 2003 & Jiang Zemin & & Ecuador, Cuba, Guyana \\
\hline 2004 & Hu Jintao & Chile, Brasil, Argentina, Cuba & $\begin{array}{l}\text { Argentina, Brasil, Venezuela (República Bolivariana de), } \\
\text { Suriname }\end{array}$ \\
\hline 2005 & Hu Jintao & México & Perú, Colombia \\
\hline 2006 & Hu Jintao & & $\begin{array}{l}\text { Venezuela (República Bolivariana de), Bolivia } \\
\text { (Estado Plurinacional de) }\end{array}$ \\
\hline 2007 & Hu Jintao & & Costa Rica \\
\hline 2008 & Hu Jintao & Perú, Costa Rica, Cuba & $\begin{array}{l}\text { Perú, Chile, México, Brasil, Venezuela } \\
\text { (República Bolivariana de) }\end{array}$ \\
\hline 2009 & Hu Jintao & & $\begin{array}{l}\text { Chile, Brasil, Venezuela (República Bolivariana de), } \\
\text { Uruguay }\end{array}$ \\
\hline 2010 & Hu Jintao & Brasil, Venezuela (República Bolivariana de), Chile ${ }^{\circ}$ & Argentina, Chile \\
\hline 2011 & $\begin{array}{l}\text { Vicepresidente } \\
\text { Xi Jinping }\end{array}$ & Cuba, Unuguay y Chile & Brasil \\
\hline 2012 & Primer Ministro Wen Jiabao & Brasil, Argentina, Uruguay y Chile & Colombia \\
\hline
\end{tabular}

Fuente: Sitio oficial del Gobierno de China, [en linea] http:J/english.gov.cn.

- Las visitas a la República Bolivariana de Venezuela y Chile fueron suspendidas a causa del terremoto acaecido en la región de Qinghai (China).

Otro país de la región que se ha visto favorecido por el acercamiento e interés del gobierno chino, ha sido Chile. Ambos países firmaron un Plan de Acción conjunto con una duración de tres años especialmente en materia relacionada con nuevas tecnologías (ingeniería antisísmica y red de monitorización de terremotos), energías alternativas e impacto medioambiental. Cabe resaltar el interés de China, de utilizar a Chile, gracias a su posición geoestratégica en la plataforma regional para desarrollar las relaciones con ASEAN y APEC buscando una gran alianza del Pacífico. Se tiene previsto crear un Comité Binacional Permanente el próximo año con una duración de 5 años para reforzar la acción conjunta bilateral y el Acuerdo de Inversiones del Tratado de Libre Comercio.

En el área específica de la cooperación al desarrollo, China ha dado a 13 países de América Latina, lo que indica su interés por ampliar su radio de influencia. La financiación ha sido a cargo del Banco de Desarrollo de China, con la firma de unos acuerdos por valor de 60.000 millones de dólares. La mayoría de los proyectos se han centrado especialmente en el desarrollo de infraestructuras, cooperación científica, temas energéticos y explotación minera y suman más de 60. (Observatorio Iberoamericano Asia-Pacífico,2010). El incremento de los proyectos de cooperación en América Latina por parte de China no tiene nada de altruismo sino todo lo contrario, son una estrategia para ampliar sus relaciones comerciales y el interés específico de explotar los vastos recursos naturales de la región, imprescindibles para su propio desarrollo económico. Parece que los objetivos de la cooperación al desarrollo de China en América Latina claramente son un instrumento clave de su política exterior con el fin de afianzar fuentes seguras, abundantes y estables de materias primas, recursos naturales, alimentos y recursos energéticos que contribuyan a mantener el ritmo de crecimiento y el proceso de industrialización y desarrollo económico chino. Ampliando sus relaciones bilaterales con muchos países latinoamericanos de renta media conseguirá ampliar nuevos mercados para incrementar los volúmenes de 
exportación e importación de bienes y servicios. China ya alcanzó a la Unión Europea como origen de las importaciones latinoamericanas, y hacia mediados de esta década podría superarla también como destino de sus exportaciones (CEPAL, 2012). Con Perú por ejemplo, la cooperación China se inicia en el año 1988 con la firma del Acuerdo Básico de Cooperación Científica y Tecnológica entre ambos gobiernos y se halla orientada a favorecer el desarrollo económico, científico y tecnológico. En el tema de ciencia y tecnología, existe un Convenio de Intercambio y Cooperación Académica desde 2005 entre el CONCYTEC y la Academia de Ciencias de China para el intercambio académico de expertos y programas de becas para estudios de maestrías y doctorados (Ministerio de Asuntos Exteriores de Perú, 2012).

Desde una perspectiva geopolítica, China quiere consolidar su posición como alternativa a los Estados Unidos, aunque no desea jugar el papel hegemónico en la región. Su interés en la región es clara, ya que sabe que sí no potencia sus relaciones con América Latina puede ser excluida ya que en la actualidad existe una amplia red de acuerdos bilaterales, regionales, triangulares o subregionales tanto con Estados Unidos como con la Unión Europea. En relación a cuestiones diplomáticas, a China le preocupa la situación con Taiwán y busca aliados y un mayor reconocimiento en este punto. Su objetivo principal es aislar al máximo al gobierno taiwanés y ganar posiciones en foros multilaterales (González Peña,2012) por medio del diálogo con diferentes agentes. Para América Latina las relaciones con China han sido muy fructíferas. Han conseguido obtener importantes inversiones directas en sectores estratégicos y créditos (Watts, 2013) a muy bajo interés para desarrollar sus infraestructuras, transportes y minería. Las buenas relaciones con el gigante asiático, garantizarán el acceso de productos latinoamericanos al mercado chino y también a otros países de la región. En este sentido se ha creado la Alianza Trans-Pacífico (TPP), también conocida como el Acuerdo Estratégico Trans-Pacífico de Asociación Económica (CEPAL, 2008), que es un acuerdo multilateral de libre comercio que incluye la reducción de barreras arancelarias y no-arancelarias para facilitar e incrementar el comercio y la inversión entre ambas regiones. Se formó originalmente por Chile, Brunei, Nueva Zelanda y Singapur en 2006. Ahora, cinco países están negociando su adhesión en el grupo: Australia, Malasia, Perú, Estados Unidos y Vietnam, y otros países, incluyendo México, Canadá y Japón, están considerando hacerlo.

\section{CONCLUSIONES}

No cabe duda que China está sobrepasando a la Unión Europea como socio inversor y donantemás importante de América Latina. Obviamente la mayor presencia la tiene China pero no hay que olvidar las inversiones y la cooperación al desarrollo de otros países asiáticos como Corea o Japón en décadas anteriores, aunque su impacto fue menor. El incremento del comercio con Asia ha reducido claramente la vulnerabilidad de América Latina y el Caribe a la desaceleración económica, que tanto han afectadoa Estados Unidos y Europa, reduciendo importantes vínculos de dependencia del pasado y creando nuevas formas de asociación comercial e importantes procesos de integración regional que refuerzan aún más su estabilidad económica y política actual.El nuevo posicionamiento geopolítico de estos gigantes económicos hacia el centro, está 
reconfigurando el mundo y está permitiendo la reinserción de América Latina en un contexto global. A pesar de estos enormes avances, aún existen fragmentaciones y grandes diferencias económicas y sociales entre países latinoamericanos.

Existe una clara división entre el Cono Sur, una región más independiente y más integrada, y América del Norte más dependiente y cercana a los regidos de Estados Unidos. Es importante que América Latina utilice esta nueva posición, las crecientes relaciones con China y el crecimiento económico de los últimos años para invertir más en sí misma,reduciendo las desigualdades y potenciando un desarrollo más sostenible.A pesar de las diferencias internas, parece que el continente latinoamericano ha sabido aprovechar la oportunidad para reconstruirse hacia fuera y reforzarse por dentro, creando importantes alianzas interregionales y transpacíficas que convertirá a América Latina en un nuevo referente en el futuro. 


\title{
The new geopolitical configuration of development cooperation in the Latin American continent: the impact of China as a new donor-investor in the region
}

\begin{abstract}
Although the European Union and the United States has been the strategic donors in the region for decades, its geopolitical interests of today have changed and Latin America is no longer a priority. In this situation and taking advantage of the economic crisis of the traditional donors and therefore a reduction in assistance, China is taking control, making the Latin American development partner communication. This comnicação analyze the changes in the forms of cooperation and new alternative structures being created in the field of development cooperation, which involved several partners in an almost horizontal triangular relationship, and China is playing an important role in a new geopolitical configuration development in the region.
\end{abstract}

KEYWORDS: Latin America. China. The geopolitics of development. Development cooperation. South-South cooperation. The triangular cooperation. 


\title{
A nova configuração geopolítica da cooperação para o desenvolvimento no continente latino-americano: o impacto da China como um novo doador-investidor na região
}

\author{
RESUMO
}

Embora a União Europeia e os Estados Unidos têm sido os doadores estratégicas na região há décadas, seus interesses geopolíticos de hoje mudaram e a América Latina deixou de ser uma prioridade. Nesta situação, e aproveitando-se da crise econômica dos doadores tradicionais e, portanto, uma redução na assistência, a China está tomando o controle, tornando a comunicação parceiro de desenvolvimento latinoamericano.Esta comnicação analisar as mudanças ocorridas nas formas de cooperação e novas estruturas alternativas que está sendo criado no domínio da cooperação para o desenvolvimento, que envolveu vários parceiros em um relacionamento triangular quase horizontal, e a China está desempenhando um papel importante em uma nova configuração geopolítica do desenvolvimento na região.

PALAVRAS-CHAVE: América Latina. China. A geopolítica do desenvolvimento. Cooperação para o desenvolvimento. Cooperação Sul-Sul. A cooperação triangular. 


\section{REFERENCIAS}

AGUDELO VILLA, H. La revolución del desarrollo. Origen y evolución de la Alianza para el Progreso. México DF: Editorial Roble. 1966.

ASHOFF, G.The Development Policy of the Federal Republic of Germany, D+C Development and Cooperation, 4, 28-56.1996.

BODEMER, K. La política alemana de cooperación para el desarrollo: de la guerra fría a la era de la globalización.Revista Española de Desarrollo y Cooperación, 3 Invierno. 1998.

BODEMER, K. La política alemana para el desarrollo de América Latina: Sus fases, tendencias y perspectivas. Revista Contribuciones, Estudios Interdisciplinarios sobre Desarrollo y Cooperación Internacional,3, Julio-Septiembre. 1985.

Die deutsche Entwicklungspolitik gegenüber Lateinamerika-eine Politik ohne Profil?.Perspektiven für die deutsch-lateinamerikanische Beziehung. Lateinamerika, Entwicklungsprozess am Wendepunkt, KlausEßer, Albert von Gleich, Institut für Lateinamerikakunde, Hamburg, 58-71. 1984.

BODEMER, K.; DRESRÜSSE, G. Nachaltige Entwicklung in Lateinamerika.Wissenschaft und Praxis im Dialog, ADLAF-Jahrestagung, Eschborn, 14-16, 63-66 y 205-212.2001.

CESARÍN, S.; MONETA, C. China y América Latina. Nuevos enfoques sobre cooperación al desarrollo. ¿Una segunda ruta de la seda?, Red de Centros de Estudios de Asia y el Pacífico de América Latina y el Caribe (REDEALAP), Buenos Aires: BID-INTAL.2005.

DENGBOLD, J.;ENDBERG, P. AID: Understanding International Development Cooperation, ZED Books: London.2013.

FISCHER, F.Informe y efectos de la Comisión Brandt. Nueva Sociedad, 55, 57-64. 1981.

GRATIUS, S.Europa y América Latina: la necesidad de un nuevo paradigma. Documentos de trabajo. Madrid: FRIDE. 2013.

GALEANO, E.Open Veins of Latin America. Five Centuries of the Pillage of a Continent, London: Serpents Tail. 2009. 
GRIFFIN, K. Foreign Aid after the Cold War.Development and Change, 22, 645685. 1991.

HALDENWANG VON, C.Integración regional en América Latina. La perspectiva de la gobernabilidad. APORTES, 195, 25-41. 2005.

HANKS, R.;STADLER, S.J. Encyclopedia of Geography Terms, Themes, and Concepts. Santa Barbara, California: ABC-CLIO. 2011.

KELLY, P. Checkerboard and Shatterbelts. The Geopolitics of South America, University of Texas Press: Austin. 1997.

MACKINDER, H.J. The Geographical Pivot of History.Democratic, Washington, DC: National Defense University Press,175-193.1996.

NUSSBAUM, M. Crear capacidades: propuestas para el desarrollo humano.Barcelona: Paidós.2012.

PEARSON, L. Partners in Development, Report of the Commission on International Development. World Bank. PallMallPress: London. 1969.

PINTOR, H. La política de cooperación de Alemania en América Latina. Revista Electrónica CAEI, Centro Argentino de Estudios Internacionales, 6, 17-29. 2006.

PINTOR, H. Una nueva era de la cooperación alemana en América Latina, Rio de Janeiro: Centro de Estudosem Geopolítica e RelaçõesInternacionais. 2013.

PINTOR, H. La cooperación alemana al desarrollo. Factores condicionantes de su transformación en la década de los noventa y su impacto en América Latina, Bern: Peter Lang. 2010.

TAFFET, J. Foreign Aid as Foreign Policy. The Alliance for Progress in Latin America, New York: Taylor Francis Group.2007.

UNCETA, K.;YOLDI, P. La cooperación al desarrollo: surgimiento y evolución histórica. Cooperación Pública Vasca. Manuales de formación $\mathrm{N}^{\circ} 1$. Vitoria Gasteiz: Servicio Central de Publicaciones del Gobierno Vasco. 2000.

ANTECEDENTES. Los Convenios de Yaundé y Arusha. Disponible en: 2014. 
BANCO INTERAMERICANO DE DESARROLLO(BID). Asia y América Latina unida a través del Acuerdo Transpacífico de Asociación Económica, 2014. : Disponible en: http://www.iadb.org/es/temas/comercio/asia-y-america-latina-unida-atraves-del-acuerdo-transpacifico-de-asociacion-economica,6121.html.Acceso:7de julio 2014.

BID y BAD analizan el futuro de la relación entre Asia y América Latina. 2014. Disponible en: $<$ http://www.iadb.org/es/temas/comercio/bid-y-bad-analizan-el-futuro-de-larelacion-entre-asia-y-america-latina,6688.html>.Acceso: 29 junio 2014.

Ten Years after the Take-off.

TakingStock of China-Latin America and the Caribbean Economic Relations. 2010. Disponible: <http://www.iadb.org/en/publications/publication- detail, 7101.html>.Acceso: 28 junio 2014.

BANCOMUNDIAL (BM). ¿What are Public Private Partnerships?2010. Disponible:<http://ppp.worldbank.org/public-privatepartnership/overview/what-are-public-private-partnerships >.Acceso: 31 mayo 2014.

BIBLIOTECA DEL CONGRESO DE CHILE. Asia-Pacífico, Brasil y China impulsan su relación con ambicioso plan de acción.2010,. Disponible:

$<$ http://asiapacifico.bcn.cl/noticias/brasil-china-plan-de-accion-20102014>.Acceso: 28 mayo 2014.

BRUNDTLAND, G. Informe para la ONU. Nuestro Futuro Común. 1987. Disponible: <http://www.un.org/es/comun/docs/?symbol=A/42/427>.Acceso: 28 de julio 2014.

CEPAL. La Unión Europea y América Latina y el Caribe. 2013.

Disponible:<http://www.eclac.cl/publicaciones/xml/0/48080/UnionEuropeaRev1. pdf $>$.Acceso:8 abril 2014.

Las relaciones económicas y comerciales entre América Latina y AsiaPacífico. El vínculo con China. 2008. Disponible: $<\underline{\text { http://www.eclac.cl/cgi- }}$ bin/getProd.asp?xml=/publicaciones/xml/3/34233/P34233.xml\&xsl=/comercio/t pl-i/p9f.xsl\&base=/comercio/tpl/top-bottom.xsl>.Acceso:15 mayo 2014.

Chinese foreign direct investment in Latin America and the Caribbean: China-Latin America cross-council taskforce.ECLAC. 2013.Disponible: <http://www.cepal.org/cgibin/getProd.asp?xml=/publicaciones/xml/1/51551/P51551.xml\&xsl=/publicacion es/ficha.xsl\&base=/publicaciones/top publicaciones.xs|>. Aceso:6 mayo 2014. 
.CEPAL destaca oportunidades de cooperación agrícola entre China y América Latina y el Caribe. ECLAC. 2013. Disponible:<http://www.eclac.cl/cgibin/getProd.asp?xml=/prensa/noticias/comunicados/9/50129/P50129.xml\&xsl=/ prensa/tpl/p6f.xsl\&base=/prensa/tpl/top-bottom.xs|>.Acceso:30 abril 2014.

CHINA TODAY. La evolución de las relaciones chino-latinoamericanas.

2012.Disponible: <http://www.chinatoday.mx/pol/content/2012-

10/24/content 491747.htm>.Acceso:11 abril 2014.

DAC.Shaping the 21st Century: The Contribution of Development Co-operation, OECD. 1996. Disponible: <http://www.oecd.org/dac/2508761.pdf>.Acceso: 29 de septiembre 2014.

GONZÁLEZPEÑA, J.M. Una aproximación a las relaciones económico-comerciales entre China y América Latina y el Caribe. Cuatro casos de estúdio.2012.

Disponible: <http://www.politica-china.org/imxd/noticias/doc/1316071208.pdf>. Acceso: 25 mayo 2014

IBEROASIA. Cooperación Asia-América Latina.Cooperación triangular. Base de Datos. 2013.Disponible:

<http://www.iberoasia.org/coop asia ibero.html?reload coolmenus\#triang>. Acceso:1 Febrero 2014.

Cooperación Asia-América Latina. Noticias de Actualidad. 2014.

Disponible:

<http://www.iberoasia.org/coop asia ibero.html?reload coolmenus\#actualidad >. Acceso:1 abril 2014.

MINISTERIO DE ASUNTOS EXTERIORES DEL PERÚ. Relaciones Bilaterales PerúRPC. Disponible: <http://www.rree.gob.pe/politicaexterior/Paginas/RelacionesBilaterales-Peru-Republica-Popular-China.aspx>.Acceso: 23 de septiembre 2014.

OBSERVATORIO IBEROAMERICANO ASIA-PACÍFICO. Boletín Observatorio № 5. Junio 2014. Disponible:

<http://www.observatorioasiapacifico.org/OBSExternalUI/pages/public/home.jsf >.Acceso: 25 mayo 2014.

\section{Boletín Observatorio № 4. Mayo}

2014. Disponible:

<http://www.observatorioasiapacifico.org/OBSExternalUI/pages/public/publicati onList.jsf?search=YES>.Acceso: 25 junio 2014. 
2014. Disponible:

<http://www.observatorioasiapacifico.org/OBSExternalUI/pages/public/publicati onList.jsf?search=YES>. Acceso: 20 mayo 2014.

Actualidad de los países en

cooperación con China. 2010. Disponible:

$<$ http://www.iberoasia.org/actualidadpaises/cn coop nov2010.pdf $>$.Acceso:5 marzo 2014.

ORGANIZACIÓN DE ESTADOS AMERICANOS. Tratados multilaterales. Tratado Interamericano de Asistencia Recíproca (TIAR). Acceso 15 de julio 2014.

OFICINA DE INFORMACIÓN. Consejo de Estado de la República Popular China. Centro de Documentación. La cooperación internacional de la República Popular China. 2011. Disponible:

<http://www.agci.cl/attachments/article/620/china.pdf>.Acceso:9 abril 2014.

ORGANIZACIÓN INTERNACIONAL DEL TRABAJO(OIT). Cooperación Sur-Sur y cooperación triangular: El camino a seguir. 2012.

Disponible:<http://www.ilo.org/wcmsp5/groups/public/---ed norm/---

relconf/documents/meetingdocument/wcms 172581.pdf>.Acceso:8 marzo 2014.

PORTAL OFICIAL DEL GOBIERNO CHINO. Disponible: <http://english.gov.cn/>. Acceso: 12 de mayo 2014.

SERVICIO EUROPEO DE ACCIÓN EXTERIOR EU-EEAS. Relaciones de la EU con América Latina, La Unión Europea y América Latina: Una asociación de actores globales. 2009. Disponible: <http://eeas.europa.eu/la/index es.htm>.Acceso: 12 de mayo, 2014.

SISTEMA ECONÓMICO LATINOAMERICANO Y DEL CARIBE. Evolución reciente de las relaciones económicas entre la República Popular China y América Latina y el Caribe. Mecanismos institucionales y de cooperación para su fortalecimento. 2010. Disponible: <http://www.iadb.org/intal/intalcdi/PE/2011/09091es.pdf>. Acceso:15 mayo 2014.

WATTS,J. China's exploitation of Latin American natural resources raises concern. The Guardian.2013.Disponible:

$<$ http://www.theguardian.com/world/2013/mar/26/china-latin-americaresources-concern>.Acceso: 20 marzo 2014. 
MORENO,L.A. Un nuevo puente entre América Latina y China. Disponible:

$<$ http://internacional.elpais.com/internacional/2014/07/15/actualidad/14054479

27 601026.html>. El País, 16 de julio, 2014. Acceso: 24 septiembre 2014.

Recebido: 20 ago. 2016

Aprovado: 03 out. 2016.

DOI: $10.3895 /$ rbpd.v6n1.5170

Como citar: PIRZKALL, H. C. P. La nueva configuración geopolítica de la cooperación al desarrollo en el

continente latinoamericano: el impacto de china como nuevo donante-inversor en la región. R. bras. Planej.

Desenv.,Curitiba, v. 6, n. 1, p. 62-83, jan./abr. 2017. Disponível em: <https://periodicos.utfpr.edu.br/rbqv>.

Acesso em: XXX.

Correspondência:

Heike Clara Pintor Pirzkall

Calle de Alberto Aguilera, 23, 28015 Madrid, España.

Direito autoral: Este artigo está licenciado sob os termos da Licença CreativeCommons-Atribuição 4.0 Internacional.

\section{(c) (1)}

